\title{
Revalorización de las aptitudes laneras de los biotipos ovinos de la región central argentina
}

\author{
Gómez, M.B. ${ }^{1}$; Castillo, M. ${ }^{\text {; }}$ Hick, M.V.H. ${ }^{2}$; Castillo, M.F. ${ }^{2}$ y Frank, E.N. ${ }^{2}$
}

'Facultad de Ciencias Veterinarias. Universidad Nacional de La Pampa. General Pico. Argentina.

${ }^{2}$ Programa SUPPRAD. Universidad Católica de Córdoba. Argentina.

\section{Palabras Clave adicionales}

Ovinicultura.

Características de lana.

Lana gruesa.

Voluminosidad.

\section{RESUMEN}

En la zona central de la República Argentina, existen explotaciones ovinas que poseen individuos con denominaciones raciales heterogéneas, cuya producción se orienta a un propósito carnicero, generando lana de calidad diversa, correspondiente en su mayoría al tipo gruesa, mayor a 28 micras. Se debería pensar en la posibilidad de realizar mezclas de lana para resaltar virtudes que las habilita para utilidades como la industria tapicera y la confección de alfombras, dicha industria requiere determinadas características en las variables de calidad de lana, tal como la voluminosidad, para que la misma sea utilizada de manera eficiente. El objetivo de este estudio fue caracterizar la producción de lana en ovinos de la región central argentina, respecto de sus variables de calidad de lana, ponderando la voluminosidad y determinar la influencia de otras variables en función de la misma. Se relevaron ovinos de la región central de la República Argentina, utilizando la técnica de muestreo denominada bola de nieve. Se empleo la metodología demográfica denominada estructura poblacional. Se alcanzo un total de 1741 ovinos en 18 unidades productivas. Se realizó una sujeción de los animales aplicando las técnicas semiológicas propias para la especie ovina. Se estableció como denominación a la apariencia general del individuo en correspondencia con características propias de estándares raciales. Durante la esquila, se pesó el vellón de cada ovino. Se tomaron en forma individual muestras de lana, en las que se determinaron, color, tipo de mecha, largo de mecha, rizos por centímetro, rinde al lavado, diámetro medio de fibra y voluminosidad. Para este estudio se dispuso de datos de 1329 ovinos. En el análisis estadístico se utilizaron medidas de resumen, frecuencias absolutas y relativas, gráficos de distribución empírica de las variables, análisis de varianza, correlación y regresión lineal múltiple. Dentro del total de lana existente el $63,94 \%$ es gruesa $>$ $28 \mathrm{\mu m}$ ), de esta el $81,29 \%$ presenta voluminosidad $>25 \mathrm{~cm}^{3} / \mathrm{g}$, que es el $52 \%$ del total de los ovinos relevados. Se infiere que la voluminosidad está relacionada con otras variables además del diámetro medio. En la región central argentina la producción ovina aporta lana en mayor proporción gruesa, la mayor parte de esta con caracteres que le confieren una voluminosidad $>25 \mathrm{~cm}^{3} / \mathrm{g}$, que es una característica requerida por la industria textil de tapicería. El recurso lanar existente, posee condiciones aptas para su aplicabilidad en la industria textil de tapicería y confección de alfombras, revalorizando así su producción, pudiéndose incrementar la misma a través de métodos simples de selección e incorporación de animales con mayor voluminosidad.

\section{Revaluation of sheep woolen skills biotypes of Argentina central region}

\section{SUMMARY}

In the central area of Argentina, there are sheep farms with heterogeneous individuals possessing heterogeneous denominations, whose production is aimed at a butcher purpose, producing wool of varying quality, which mostly corresponds to a heavy type, greater than 28 microns. The possibility of wool blends to highlight virtues that enables them to profit as the upholstery industry and carpet weaving should be considered, as the industry requires certain characteristics in wool quality variables, such as bulk, so that it is used efficiently. The aim of this study was to characterize the production of sheep wool in central Argentina, for their wool quality variables, considering the bulkiness and determining the influence of other variables depending on its variability. Sheep in the central region of Argentina were surveyed using sampling technique called snowball, using a demographic methodology called population structure. A total of 1741 sheep was reached in 18 production units. The animals were held by applying the semiotic techniques of common use for ovine animals. The overall appearance of the individual in correspondence with racial own standard features was denominated. During shearing, the wool of each sheep was weighed. Individual samples of wool, in which color, type of wick, staple length, crimps per centimeter, yields wash, average fiber diameter, bulk were simultaneously determined as they were taken. For this study the data of 1329 sheep was used. The statistical analysis used summary measures, absolute and relative frequencies, graphics empirical distribution of variables, analysis of variance, correlation and multiple linear regression. From the total existing, a $63.94 \%$ of wool 
is thick $(>28 \mu \mathrm{m}), 81.29 \%$ of these showed bulkiness $>25 \mathrm{~cm}^{3} / \mathrm{g}$, corresponding to a $52 \%$ of all sheep relieved. Bulkiness is inferred to be influenced by other variables apart from mean diameter. The Argentinian central region sheep wool production supplies a greater proportion of thick wool, most of which presents bulkiness providing characters $>25 \mathrm{~cm}^{3} / \mathrm{g}$, which is a required characteristic by the textile industry upholstery. The existing wool resort has suitable conditions for its applicability in the textile industry and enriching its production, being able to increase it through simple methods of selection and the incorporation of animals showing a greater bulkiness.

\section{INTRODUCCIÓN}

Desde tiempos de la colonia, en la Región pampeana, centro y norte del territorio argentino el aprovechamiento del ovino registra procesos de colonización, poblamiento y absorción. Algunas poblaciones se mantuvieron inalteradas, otras fueron influenciadas notoriamente por las continuas introducciones y quedaron muchas en una situación intermedia (Helman, 1965).

En la zona central de la República Argentina, existen explotaciones ovinas que poseen individuos con denominaciones raciales heterogéneas, cuya producción se orienta a un propósito carnicero, lo cual es acertado acorde al objetivo productivo y a las oportunidades futuras de la región, generando lana de calidad diversa, correspondiente en su mayoría al tipo gruesa, mayor a 28 micras. Se debería pensar en la posibilidad de realizar mezclas de lana para resaltar virtudes que las habilita para utilidades como la industria tapicera y la confección de alfombras (Gómez et al., 2016). Dicha industria, requiere determinadas características en las variables de calidad de lana, para que la misma sea utilizada de manera eficiente, tal como demostraron Carnaby y Elliot (1980) en estudios de procesamiento en referencia a variables tal como la voluminosidad. Elliot en 1984, estimo que una medida de voluminosidad mayor a $25 \mathrm{~cm}^{3} / \mathrm{g}$, debería brindar un precio diferencial. De hecho en Nueva Zelanda cada vez mas lana se negocia con respecto a la medición objetiva de parámetros como indicadores de rendimiento de procesamiento. Los procedimientos de pruebas de pre-venta implican medidas tomadas de lana sucia prensada y la certificación de prueba estándar incluye mediciones de rendimiento, contenido de materia vegetal y diámetro de la fibra, con una serie de mediciones adicionales disponibles bajo petición, entre ellas, la voluminosidad (Sumner et al., 2001), que además posee alta heredabilidad y está controlada por relativamente pocos genes (Sumner et al., 1995; Wuliji et al., 1995).

El conocimiento de las características de la lana autóctona, posibilitara el estímulo necesario para desarrollar la actividad y hacer sustentable el sistema productivo, reconociendo sus beneficios para revalorizarla (Gómez et al., 2012).

El objetivo de este estudio es caracterizar la producción de lana en ovinos de la región central argentina, respecto de sus variables de calidad de lana, ponderando la voluminosidad y determinar la influencia de otras características en función de la misma.

\section{MATERIALES Y MÉTODOS}

Se relevaron ovinos de la región central de la República Argentina, que comprende territorio del oeste de la provincia de Córdoba, noreste de San Luis y norte de La Pampa, conformando una zona semiárida. El área en estudio se caracteriza por ser agrícola-ganadera.

Cada grupo de ovinos pertenecientes a un establecimiento fue definido como la unidad productiva ovina o bien majada. El relevamiento de estos animales, consistió en la utilización de la técnica de muestreo denominada bola de nieve (Cochran, 1974; Frank, 2001; Sandoval Casilimas, 2002; Hick y Frank, 2013).

Se utilizo la metodología demográfica denominada estructura poblacional que fue propuesta y utilizada en camélidos (Lauvergne et al., 1997), revisada y descripta para poblaciones de rumiantes menores productores de fibra (Hick et al., 2012; Hick y Frank, 2013). Se utilizó la misma, mediante una correcta recopilación de información registrada en planillas.

Se estableció como denominación a la apariencia general del individuo en correspondencia con características propias de estándares raciales: Corriedale, Criolla, Hampshire Down, Karacul, Pampinta, Texel y Merino.

En todos los casos se realizó una sujeción de los animales aplicando las técnicas semiológicas propias para la especie ovina, que generan el bienestar tanto del animal como de los operarios.

Se alcanzo un total de 1741 ovinos en 18 unidades productivas.

Durante la esquila, se pesó cada vellón. Se tomaron en forma individual muestras de lana, de la región del flanco izquierdo con una tijera de esquila estándar, se pesaron $30 \mathrm{~g}$ y luego se acondicionaron en bolsas de nylon a las que se les quitó el aire y rotularon para ser remitidas al laboratorio.

El análisis de las muestras se realizó en el Laboratorio de Fibras Animales de la Red SUPPRAD con sede en la Facultad de Ciencias Agropecuarias de la Universidad Católica de Córdoba, donde se contó con los diferentes gabinetes de almacenamiento de muestras, lavado, acondicionamiento y clasificación. Las muestras fueron procesadas a medida que se iban realizando los relevamientos.

Se determinaron, color (CM), tipo de mecha (TM), largo de mecha (LM), rizado (RZ), rendimiento al lavado (RLV), diámetro medio de fibra (DM) y voluminosidad (BK), (Bigham et al., 1984).

El procesamiento de la muestra se realizó mediante la extracción del grupo de mechas y luego su acondicionamiento durante 15 minutos, para clasificar las mechas por los criterios de calidad de lana. En sucio se determinaron, CM, TM representada por capa intermedia (CI), doble capa (DC), hemilustre (HL), lustre (L) y simple capa (SC), a partir de lo expuesto por Rougeot (1982) y adaptado por Hick et al. (2007a y 2007b), LM en cm y RZ en rizos/cm, para los que se utilizo una regla graduada (para rizos en pulgadas y luego se calculo el valor que corresponde por $\mathrm{cm}$ ). 
El lavado fue realizado mediante el pasaje por 4 bateas con solución de agua y detergente decreciente con el pH corregido. En cada batea se realizó una inmersión de 5 minutos y luego un escurrido antes del siguiente pasaje. Finalmente, fueron secadas en una estufa a $40{ }^{\circ} \mathrm{C}$ (Ryder and Stephenson, 1968; Lamb, 1998; Frank, Hick y Adot, 2012). En mechas limpias lavadas y secadas, se determino DM en $\mu \mathrm{m}$, se realizó en la mecha completa, con un microproyector lanámetro a 500x en base la normativa ITWO 80-3, adaptada al establecer un número mínimo de observaciones o conteos para alcanzar un error deseado (Lamb, 1998; Frank, Hick, Prieto y Castillo, 2009).

BK en $\mathrm{cm}^{3} / \mathrm{g}$, se determinó mediante la utilización de un bulkómetro de bronce, que consta de un pistón con un cilindro el cual lleva una escala graduada $\mathrm{mm}$ capaz de medir el volumen de la muestra después de una compresión de prueba/recuperación (WRONZ). nos.

Para este estudio se dispuso de datos de 1329 ovi-

En el análisis estadístico de la información se utilizaron medidas de resumen, frecuencias absolutas y relativas, gráficos de distribución empírica de las variables, análisis de varianza, análisis de correlación lineal de Pearson y de Spearman y regresión lineal múltiple.

\section{RESULTADOS Y DISCUSIÓN}

Dentro del total de lana existente, el 63,94\% es gruesa (> $28 \mu \mathrm{m})$, en concordancia con lo demostrado por Gómez et al. (2016), de esta el $81,29 \%$ presenta BK $>25 \mathrm{~cm}^{3} / \mathrm{g}$, correspondiente al $52 \%$ del total de los ovinos relevados (tabla I).

El $25,82 \%$ de los ovinos poseen lana con $\mathrm{DM} \leq 28$ $\mu \mathrm{m}$ y tienen también BK $>25$ en $\mathrm{cm}^{3} / \mathrm{g}$ (tabla I). Con esta evidencia, se infiere que BK está relacionada con otras variables además del DM.

Como fue demostrado por Chaudri et al. (1968), existen variaciones en el volumen de compresión de los diferentes tipos de lana, que es afectado de forma significativa por las características del rizado (forma y frecuencia, que juntas son altamente significativas) y el diámetro de fibra. Fue confirmado por Sumner et al. (2001), tras varios ensayos previos, que las medias del diámetro de la fibra y la curvatura de la misma, tienen una fuerte relación con la voluminosidad $\left(R^{2}>65\right)$ y pueden ser utilizadas para clasificar las ovejas de lana gruesa independientemente de su genotipo.

Dados estos antecedentes y la observación de otras características que coexisten con las ya mencionadas en la fibra de lana, se analizaron las mismas como variables, para examinar indicios de su relación con la voluminosidad.

En análisis de correlación en relación a estas variantes dentro de los criterios de clasificación de lana que se utilizaron, se observo que DM y BK presentan asociación lineal y significativa $(r=0,08 ; \mathrm{p}<0,01)$, así también RZ y BK $(r=0,24 ; \mathrm{p}<0,001)$ y LM y BK $(\mathrm{r}=$ $-0,27 ; \mathrm{p}<0,001)$.

Tabla I. Características de lana en ovinos de la región central argentina (Characteristics of wool in sheep of central Argentina)

\begin{tabular}{|c|c|c|c|c|c|c|c|c|c|c|c|}
\hline \multirow{2}{*}{\multicolumn{2}{|c|}{ Criterio }} & \multirow{2}{*}{ TM } & \multicolumn{8}{|c|}{ Denominación } & \multirow[b]{2}{*}{ E.E. } \\
\hline & & & Corriedale & Criolla & H. Down & Karacul & Pampinta & Texel & Merino & Total & \\
\hline \multirow{9}{*}{ 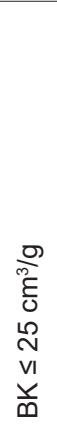 } & \multirow{4}{*}{$\begin{array}{l}\text { E } \\
\text { D } \\
\stackrel{\infty}{v} \\
\text { v1 } \\
\sum\end{array}$} & Cl-DC & 0,15 & 0,14 & 0,00 & 0,07 & 0,00 & 0,00 & 0,00 & & 0,00 \\
\hline & & HL-L & 0,45 & 0,00 & 0,07 & 0,00 & 0,00 & 0,00 & 0,22 & & 0,01 \\
\hline & & SC & 1,65 & 0,30 & 0,30 & 0,07 & 1,28 & 0,00 & 5,42 & & 0,04 \\
\hline & & & 2,25 & 0,44 & 0,37 & 0,14 & 1,28 & 0,00 & 5,64 & 10,12 & 0,04 \\
\hline & \multirow{4}{*}{$\begin{array}{l}\underline{\xi} \\
\infty \\
\stackrel{\infty}{N} \\
\Lambda \\
\sum\end{array}$} & Cl-DC & 0,60 & 2,78 & 0,07 & 0,22 & 0,07 & 0,00 & 0,00 & & 0,03 \\
\hline & & HL-L & 1,43 & 0,45 & 0,83 & 0,00 & 0,30 & 0,00 & 0,00 & & 0,01 \\
\hline & & SC & 2,11 & 0,30 & 0,98 & 0,00 & 1,80 & 0,00 & 0,00 & & 0,02 \\
\hline & & & 4,14 & 3,53 & 1,88 & 0,22 & 2,17 & 0,00 & 0,00 & 11,94 & 0,04 \\
\hline & Subtotal & & 6,39 & 3,97 & 2,25 & 0,36 & 3,45 & 0,00 & 5,64 & 22,06 & 0,05 \\
\hline \multirow{10}{*}{$\begin{array}{l}\frac{0}{0} \\
\stackrel{0}{0} \\
0 \\
\stackrel{N}{N} \\
\hat{~} \\
\text { m }\end{array}$} & \multirow{4}{*}{ 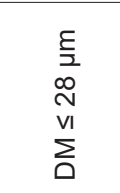 } & Cl-DC & 0,37 & 0,37 & 0,00 & 0,07 & 000 & 0,00 & 0,00 & & 0,00 \\
\hline & & HL-L & 0,97 & 0,07 & 0,07 & 0,00 & 0,07 & 0,00 & 0,07 & & 0,01 \\
\hline & & SC & 12,41 & 4,13 & 1,58 & 0,00 & 2,93 & 0,6 & 2,11 & & 0,13 \\
\hline & & & 13,75 & 4,57 & 1,65 & 0,07 & 3,00 & 0,60 & 2,18 & 25,82 & 0,15 \\
\hline & \multirow{4}{*}{$\begin{array}{l}\frac{\xi}{2} \\
\stackrel{\infty}{N} \\
\wedge \\
\sum \\
\sum\end{array}$} & Cl-DC & 1,13 & 2,49 & 0,22 & 0,00 & 0,00 & 0,00 & 0,00 & & 0,02 \\
\hline & & HL-L & 2,56 & 1,05 & 1,28 & 0,00 & 0,15 & 0,38 & 0,00 & & 0,03 \\
\hline & & SC & 14,97 & 9,03 & 14,3 & 0,30 & 2,11 & 2,03 & 0,00 & & 0,15 \\
\hline & & & 18,66 & 12,57 & 15,80 & 0,30 & 2,26 & 2,41 & 0,00 & 52,00 & 0,18 \\
\hline & \multicolumn{2}{|l|}{ Subtotal } & 32,41 & 17,14 & 17,45 & 0,37 & 5,26 & 3,01 & 2,18 & 77,82 & 0,30 \\
\hline & \multicolumn{2}{|l|}{ Total } & 38,80 & 21,11 & 19,70 & 0,73 & 8,71 & 3,01 & 7,82 & 99,88 & 0,33 \\
\hline
\end{tabular}

TM: tipo de mecha; BK: voluminosidad; DM: diámetro medio; Cl: capa intermedia; DC: doble capa; HL: hemilustre; L: Iustre; SC: simple capa. 
Tabla II. Análisis de varianza de peso de vellón en $\mathrm{kg}$ para denominaciones ovinas con lana de DM $>28 \mu \mathrm{m}$ y BK $>25 \mathrm{~cm}^{3} / \mathrm{g}$ (Analysis of variance of fleece weight in $\mathrm{kg}$ for sheep denominations with wool of DM $>28 \mu \mathrm{m}$ and $\mathrm{BK}>25 \mathrm{~cm}^{3} / \mathrm{g}$ ).

\begin{tabular}{lccc}
\hline Denominación & Media & E. E. & \\
\hline Criolla & 2,89 & 0,15 & A \\
H. Down & 3,77 & 0,12 & B \\
Corriedale & 3,99 & 0,12 & B \\
Pampinta & 4,53 & 0,20 & BC \\
Texel & 4,83 & 0,19 & C \\
Karacul & 4,90 & 0,64 & C \\
\hline
\end{tabular}

Medias con una letra común no son significativamente diferentes $(p \leq 0,05)$.

Animales con TM simple capa tienen asociación positiva y significativa con lana de $\mathrm{BK}>25 \mathrm{~cm}^{3} / \mathrm{g}\left(\mathrm{r}_{\mathrm{s}}=\right.$ $0,72 ; \mathrm{p}<0,001)$, tal como animales con TM hemilustre $\mathrm{y}$ lustre $\left(r_{s}=0,43 ; p<0,05\right)$, en animales con TM capa intermedia y doble capa la asociación no es significativa $(\mathrm{p}>0,05)$.

El segmento de interés en este estudio es lana con $\mathrm{DM}>28 \mu \mathrm{m}$, dentro de la cual resulto:

Lana con $\mathrm{BK} \leq 25 \mathrm{~cm}^{3} / \mathrm{g}$, presenta desvío del diámetro medio (StDev) más extenso y una media de RZ de 2,57 (D.E.: 0,90, E.E.: 0,07) respecto a aquella que presenta $\mathrm{BK}>25 \mathrm{~cm}^{3} / \mathrm{g}$ donde RZ es 3,29 (D.E.: 0,94, E.E.: 0,04) y la distribución de la variable tiene mayor frecuencia en torno a este y a valores superiores.

Lana con $\mathrm{BK}>25 \mathrm{~cm}^{3} / \mathrm{g}$, referente a TM, posee mayor proporción de simple capa (SC), respecto a aquella con $\mathrm{BK} \leq 25 \mathrm{~cm}^{3} / \mathrm{g}$ (tabla I).

Lana con $B K \leq 25 \mathrm{~cm}^{3} / \mathrm{g}$, tiene una media de LM de $11,82 \mathrm{~cm}$ (D.E.: 7,15, E.E.: 0,60), mientras que en la que posee $\mathrm{BK}>25 \mathrm{~cm}^{3} / \mathrm{g}$ la misma es 6,69 (D.E.: 3,99, E.E.: $0,16)$ y en esta la distribución de la variable es menos frecuente hacia valores muy superiores al mencionado (figura 1 y 2).

En base a estas evidencias, en lana gruesa $>28 \mu \mathrm{m}$ se realizo la regresión lineal múltiple en la que se incorporaron las variables antes consideradas.

\section{$\mathrm{R}^{2} 0,44$}

$\mathrm{BK}=20,32+0,09 \times \mathrm{DM}-0,11 \times \mathrm{StDev}+1,31 \times \mathrm{RZ}+$ $1,11 \times \mathrm{TM}-0,14 \times \mathrm{LM}$

Esto indica que el $44 \%$ de la variabilidad de BK es explicada por las variables regresoras, con un $\mathrm{p}<0,05$. Este coeficiente de regresión obtenido con los datos de lana gruesa de los biotipos ovinos de la región central argentina, es menor al hallado por otros autores (Chaudri et al.; 1968; Sumner et al., 2001) en otros ambientes y con otros rangos de variación de los caracteres. Además en este estudio fueron incorporadas más variables.

En los ovinos con lana de $\mathrm{DM}>28 \mu \mathrm{m}$ y $\mathrm{BK}>25$ $\mathrm{cm}^{3} / \mathrm{g}$, la denominación Criolla es la de menor peso de vellón, con una media de $2,89 \mathrm{~kg}$ y presenta diferencias significativas con el resto; Texel y Karacul que tienen los mayores pesos, con una media de $4,83 \mathrm{~kg} \mathrm{y} \mathrm{4,90} \mathrm{kg}$ respectivamente, no son significativamente diferentes entre sí, $\mathrm{p}<0,05$ (tabla II).

El RLV sin embargo, es mayor en la Criolla con una media de 72,95\% (tabla III).

\section{CONCLUSIONES}

En la región central argentina la producción ovina aporta lana en su mayor proporción de tipo gruesa, que en su mayoría presenta caracteres que le confieren una voluminosidad mayor a $25 \mathrm{~cm}^{3} / \mathrm{g}$, característica

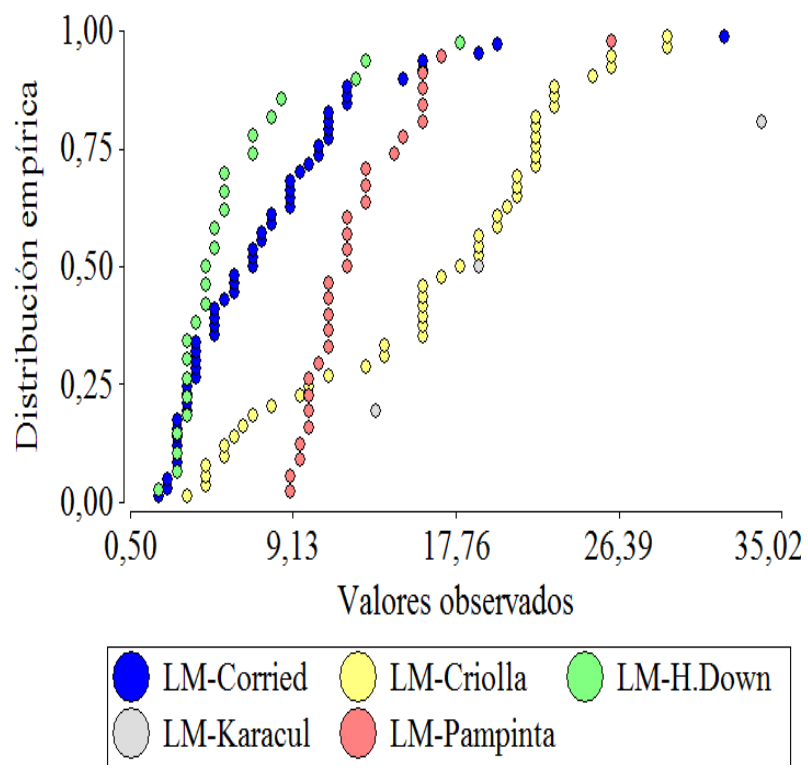

LM: largo de mecha.

Figura 1. Distribución de LM en lana con DM $>28 \mu \mathrm{m}$ $\mathrm{y} \mathrm{BK} \leq 25 \mathrm{~cm} 3 / \mathrm{g}$ (Distribution of LM in wool with $\mathrm{DM}>28 \mu \mathrm{m}$ and $\mathrm{BK} \leq 25 \mathrm{~cm} 3 / \mathrm{g}$ ).

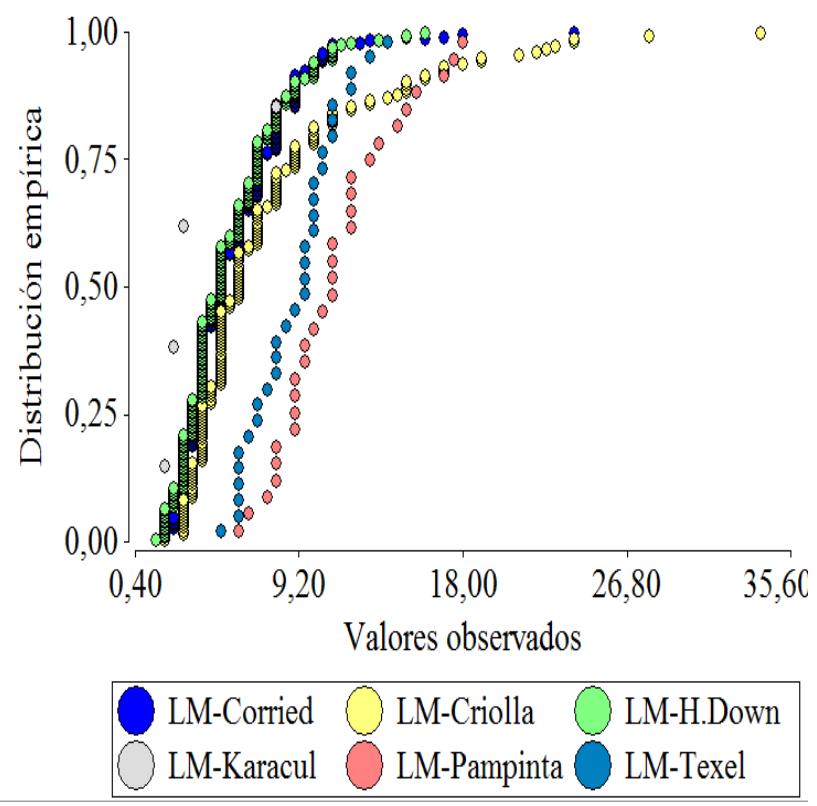

LM: largo de mecha.

Figura 2. Distribución de LM en lana con DM $>8 \mu \mathrm{m}$ $\mathrm{y} \mathrm{BK}>25 \mathrm{~cm}^{3} / \mathrm{g}$ (Distribution of LM in wool with DM $>28 \mu \mathrm{m}$ and $\mathrm{BK}>25 \mathrm{~cm}^{3} / \mathrm{g}$ ). 
Tabla III. Rednimiento al lavado en ovinos con lana de DM $>28 \mu \mathrm{m}$ y BK $>25 \mathrm{~cm}^{3} / \mathrm{g}$ (Yield at washing in sheep with DM wool $>28 \mu \mathrm{m}$ and $\mathrm{BK}>25 \mathrm{~cm}^{3} / \mathrm{g}$ ).

\begin{tabular}{lcccc}
\hline Denominación & Media & D. E. & Mín & Máx \\
\hline Corriedale & 64,01 & 12,54 & 33,24 & 96,83 \\
Pampinta & 66,11 & 8,64 & 41,41 & 82,70 \\
Texel & 66,56 & 8,27 & 48,31 & 87,26 \\
Karacul & 68,02 & 8,55 & 56,02 & 76,26 \\
H. Down & 68,32 & 11,32 & 45,07 & 114,09 \\
\hline Criolla & 72,95 & 11,76 & 38,56 & 99,49
\end{tabular}

requerida por la industria textil de tapicería y para la confección de alfombras. Estos atributos deberían ser identificados y resaltados, a través de maniobras simples, posibles de realizar en cada unidad productiva, tales como selección de animales mediante la inspección de la lana, considerando el rizado, tipo de mecha y largo de la misma, que están asociados a una voluminosidad superior, revalorizando el producto, por lo cual es necesario considerar la voluminosidad como parámetro de calidad y para esto incluir su medición en el sistema de clasificación para certificación.

\section{AGRADECIMIENTOS}

El presente estudio fue realizado con recursos aportados por la Facultad de Ciencias Veterinarias de la Universidad Nacional de La Pampa y del Programa SUPPRAD de la Universidad Católica de Córdoba.

\section{BIBLIOGRAFÍA}

Bigham, M.L.; Sumner, R.M.W. and Cox, N.R. 1984. The measurement and sources of variation of loose wool bulk in Romney, Coopworth and Perendale breeds. New Zeal J Agr Res, 12: 23-28.

Carnaby, G.A. and Elliott, K.H. 1980. Bulk. A wool trait of importance to the carpet industry. Proceedings of the New Zealand Society of Animal Production, 40: 196-204.

Chaudri, M.A. and Whiteley, K.J. 1968. The influence of natural variations in fiber properties on the bulk compression of wool. Textile Res J, 38: 897-906.

Cochran, W.G. 1974. Técnicas de muestreo. Compañía Editorial Continental, México D.F. 507 pp.

Elliot, K.H. 1984. Price premiums for high-bulk Perendale wools. Proceedings of the New Zeland Society of Animal Production, 44: 41-44.

Frank, E.N. 2001. Descripción y análisis de la segregación de fenotipos de color y tipos de vellón en llamas argentinas. Tesis de doctorado (UBA).

Frank, E.N.; Hick, M.V.H.; Prieto, A. y Castillo, M.F. 2009. Metodología de identificación cualitativa y cuantitativa de fibras textiles naturales. Serie Documentos Internos SUPPRAD N ${ }^{\circ} 1$, Red SUPPRAD. pp. 14. (23/06/2016).

Frank, E.N.; Hick, M.V.H. and Adot, O. 2012. Determination of dehairing tactile attributes with different Llama fleecetypes. Arch Sci, 12: 294-312.

Gómez, M.B.; Castillo, M.; Aguirre, S.I.; Hick, M.V.H.; Prieto, A.; Castillo, M.F.; Meglia, G.E. y Frank, E.N. 2012. Determinación preliminar del potencial de la calidad de la lana de majadas ovinas en la Región Norte de la Provincia de La Pampa. Cienc Vet, 14: 6-10.
Gómez, M.B.; Castillo, M.; Aguirre, S.I.; Díaz, W.; Vargas, M.; Hick, M.V.H.; Castillo, M.F. y Frank, E.N. 2016. Caracterización de la estructura de los rebaños ovinos del norte de la provincia de La Pampa. Rev Asoc Latinoam Prod Anim, 24: 1022-1301.

Helman, M.B. 1965. Ovinotecnia. El Ateneo. Buenos Aires.

Hick, M.V.H.; Frank, E.N.; Prieto, A.; Gorocito, C.; Callegaris, C.M.; Baigorria Herrera, L. y Pons, F. 2007b. Determinación del potencial textil de majadas ovinas de la pampa de Olaen, provincia de Córdoba. En: V Congreso ALEPRYCS. Mendoza, 2-4 Mayo. Resumen Menorías. pp. 93.

Hick, M.V.H.; Frank, E.N.; Prieto, A.; Gorocito, L.C.; Savid, M.D.; González, D.F. e Isaia A., M.B. 2007a. Determinación del potencial textil de majadas ovinas de las Sierras Centrales, provincia de Córdoba. Rev Arg Prod Anim, 27: 360-361.

Hick,M.V.H.; Frank, E.N.; Prieto, A.; Ahumada, M.R.y Castillo, M.F. 2012. Alcances de la metodología de Estructura Poblacional. Hick, M.V.H. y E.N. Frank (Eds). Documento Interno SUPPRAD № 3 (23/06/2016).

Hick, M.V.H. y Frank, E.N. 2013. Descripción y alcances de la utilización de la metodología de estructura poblacional en rumiantes menores productores de fibra. En Memorias XXIII Reunión Latinoamericana de Producción Animal y IV Congreso Internacional de Producción Animal Tropical. pp. 2622-2633.

Lamb, P. 1998. Fibre Metrology of Wool and its Applicability to Alpaca. In: L. D. Brash and I. M. Davison. 1998 (Eds.). Fibre Science and technology: Lessons from the wool industry. Proceeding of a Conference held at CSIRO. Animal Production Prospect, NSW. Australian. pp. 13-20.

Lauvergne, J.J.; Frank, E.N.yHick, M.V.H. 1997. Dinámica de la población de Camélidos. En: Primer infirme de Avance del Proyecto SUPPREME (UE-DGXII-INCO). Work-package C. pp. 2-3.

Rougeot, J. 1982. Evolution de la toison en relation avec les caracteristiques textiles. Bull Scient ITF, 1: 41-52.

Ryder, M.L. and Stephenson, S.K. 1968. Wool growth. Academic Press. New York.

Sandoval Casilimas, C.A. 2002. Investigación cualitativa. En: Briones, G. (Ed.) Especialización en teoría, métodos y técnicas de investigación social. ICFES, Colombia. En: http://es.scribd.com/doc/7634389/ Casilimas-Sandoval-Investigacion-Cualitativa (23/06/2016).

Sumner, R.M.W.; Clarke, J.N. and Cullen, N.G. 1995. Responses to selection for and against loose wool bulk and the associated effect on clean fleece weight and objective fleece characteristics in yearling Perendale and Cheviot sheep.In: Bona M. and Grupallo, G. (Eds.). Proceedings $9^{\text {th }}$ International Wool Textile Research Conference. Biella. Italy.

Sumner, R.M.W and Upsdell, M.P. 2001. Factors associated with the prediction of core bulk from fibre diameter and fibre curvature of individual fleeces. Wool Tech Sheep Breed, 49: 29-41.

Wuliii T.; Montgomery, G.M.; Dodds, K.G.; Andrews, R.N.; Beattie, A.E.; Turner, P.R. and Rogers, J. 1995. Establishing a flock for gene mapping in wool traits. Proceedings New Zeland Society Animal Production. Vol. 55: 285-288. 